COLORECTAL CANCER

\title{
Whole body positron emission tomography/ computed tomography (PET/CT) tumour staging with integrated PET/CT colonography: technical feasibility and first experiences in patients with colorectal cancer
}

\author{
P Veit, C Kühle, T Beyer, H Kuehl, C U Herborn, G Börsch, H Stergar, J Barkhausen, \\ A Bockisch, G Antoch
}

See end of article for authors' affiliations

Correspondence to: Dr P Veit, Department of Diagnostic and

Interventional Radiology,

University Hospital of

Essen, Hufelandstrasse 55,

45122 Essen, Germany;

patrick.veit@uni-essen.de

Revised version received 10 March 2005

Accepted for publication

29 March 2005

Published online first

14 June 2005

\begin{abstract}
Aim: The aim of this study was to implement an imaging protocol for positron emission tomography/ computed tomography (PET/CT) colonography and to combine this protocol with whole body PET/CT tumour staging for a single whole body examination for routine clinical use.

Subjects and methods: A whole body PET/CT protocol for tumour staging and a protocol for PET/CT colonography were integrated into one examination. Fourteen prospective patients with suspected colorectal cancer underwent whole body PET/CT after aqueous bowel distension and pharmacological bowel relaxation. Colonoscopy and histopathology served as the standards of reference in all patients. Results: The modified PET/CT examination detected all but one lesion in the colon. One additional lesion was detected in a patient with incomplete colonoscopy due to high grade luminal stenosis. One polyp with malignant conversion was identified with the modified PET/CT protocol. PET/CT colonography proved accurate in local lymph node staging and staged nine out of 11 patients correctly. Six additional extracolonic tumour sites were detected based on the whole body staging approach.

Conclusion: Whole body PET/CT with integrated colonography is technically feasible for whole body staging in patients with colorectal cancer. Based on these initial diagnostic experiences, this integrated protocol may be of substantial benefit in staging patients with colorectal cancer, focusing on patients with incomplete colonoscopy and those with small synchronous bowel lesions.
\end{abstract}

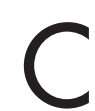
olorectal cancer is a leading cause of death in Western countries and its incidence has increased over the past 20 years. ${ }^{1-3}$ Accurate tumour staging is a prerequisite for therapy planning and for successful therapy of patients suffering from colorectal cancer. ${ }^{4-8}$ In the clinical setting, conventional colonoscopy is performed for tumour detection, tissue sampling, and to exclude synchronous lesions. Usually colonoscopy is performed in conjunction with non-invasive imaging techniques to assess potential metastases to lymph nodes and distant organs. ${ }^{6} 89$

Preoperative contrast enhanced computed tomography (CT) in different technical variations has emerged as one of the most frequently used tools for staging of colorectal

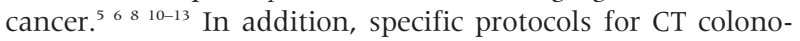
graphy offer visualisation of the primary tumour combined with evaluation of adjacent and distant abdominal organs for potential metastases. ${ }^{14}$ A limitation of CT and other radiological imaging procedures is their lack of functional data, which may render determination of lesion size, potential infiltration of adjacent organs, or involvement of locoregional lymph nodes difficult. ${ }^{15}$

$\left[{ }^{18} \mathrm{~F}\right]$-Fluoro-2-deoxy-D-glucose (FDG)-positron emission tomography (PET), on the other hand, is highly accurate when staging primary and recurrent colorectal cancer. ${ }^{16-21}$ Depending on the study and patient population, sensitivities of $93-100 \%$ were found and specificities for tumour detection and characterisation ranged from $43 \%$ to $98 \%$. PET imaging has been shown to be superior to conventional CT staging when assessing patients with colon cancer for distant metastases. ${ }^{19}$ However, curtailed anatomical information provided by FDG-PET often renders accurate localisation of metastatic lesions difficult. ${ }^{22}$ Dual modality PET/CT scanners provide accurately fused morphological and functional imaging within a single examination. Therefore, the major limitation of FDG-PET, lack of anatomical information, can be overcome. ${ }^{23}{ }^{24}$ In addition, functional data are provided by PET/CT compared with CT alone. A variety of studies have demonstrated this hybrid imaging tool to be superior to CT alone and PET alone for lesion detection and characterisation when staging patients with different malignancies and those with colorectal cancer. ${ }^{15}{ }^{22} 25-27$ Thus a combined whole body PET/CT colonography approach may serve as an attractive alternative to a multistep multimodality workup comprising CT staging, PET, and conventional colonoscopy. Although this approach may not be designed to replace optical colonoscopy, patients with incomplete colonoscopy or those with small synchronous bowel tumours may benefit from such an optimised PET/CT protocol.

Thus the aim of this prospective study was to develop a protocol for an optimised whole body PET/CT staging examination, including virtual colonography, and to assess the technical feasibility of such a protocol.

Abbreviations: $\mathrm{PET}$, positron emission tomography; $\mathrm{CT}$, computed tomography; FDG, [ $\left.{ }^{18} \mathrm{~F}\right]$-fluoro-2-deoxy-D-glucose; 3D-MPR, three dimensional-multiplanar reformatting; SUV, standard uptake value 


\section{SUBJECTS AND METHODS \\ Patients}

Fourteen consecutive patients (mean age 76 years (range 5692); 11 females, three males) were enrolled in the study. All patients were admitted to the hospital because of bright red blood per rectum (four patients), positive faecal occult blood test (three patients), altered bowel habits (two patients), anaemia of unknown cause (three patients), and severe and long lasting abdominal pain (two patients). None of the patients suffered from inflammatory bowel disease. All patients were referred for PET/CT staging based on suspicion of colorectal cancer after having undergone conventional colonoscopy one day previously, with biopsy of suspicious bowel lesions.

Colonoscopy was incomplete in one patient due to high grade stenosis of the colonic lumen by a large tumour. Eleven patients were operated on after tumour staging; three patients received chemotherapy. Informed consent was obtained from all patients before enrolling in this prospective study. The study was performed in accordance with the regulations of the local ethics committee.

\section{PET/CT imaging procedure}

Dual modality imaging was performed with a biograph PET/ CT system (Siemens Medical Solutions, Hoffman Estates, Illinois, USA). The system is based on two components: a dual slice CT scanner (Somatom Emotion, Siemens Medical Solutions, Forchheim, Germany) and a full ring PET tomograph (ECAT HR+; Siemens Medical Solutions). The PET system has an axial field of view of $15.5 \mathrm{~cm}$ per bed position and an in plane spatial resolution of $4.6 \mathrm{~mm}$. The system acquires the CT first, followed by PET. After examination, CT and PET data sets can be viewed separately or in fused mode on a commercially available computer workstation (Syngo Software; Siemens Medical Solutions, Erlangen, Germany).

Two litres of a bowel cleansing solution containing polyethylene glycol-electrolytes (PEG-ES, GoLYTELY; Braintree Laboratories Inc., Braintree, Massachusetts, USA) was administered to all patients for bowel cleansing. All patients had been instructed to fast for a minimum of six hours prior to the examination. Blood glucose levels were found to be in the normal range prior to FDG injection by blood sampling. In all patients, $330 \mathrm{MBq}$ of FDG were administered 60 minutes prior to the PET/CT examination. In addition, $1500 \mathrm{ml}$ of a negative oral contrast agent were given within the FDG uptake time for small bowel distension. ${ }^{28}$

Acquisition of whole body PET/CT with a field of view from the skull to the upper thighs was divided into two parts. Data from the upper body regions (base of skull to diaphragm) were acquired first in a caudocranial direction with the patient in the supine position using a standardised breathing protocol. ${ }^{29}$ Image acquisition was performed with $110 \mathrm{mAs}$, $120 \mathrm{kV}, 5 \mathrm{~mm}$ slice thickness and a $2.4 \mathrm{~mm}$ incremental reconstruction, and $60 \mathrm{ml}$ of iodinated contrast agent (Xenetix 300, Guerbet GmbH, Sulzbach, Germany). PET data were acquired with the same field of view as CT (base of skull to diaphragm). In the second imaging part of the study, all patients received pharmacological bowel relaxation (20 $\mathrm{mg}$ of $\mathrm{N}$-butylscopalamine (Buscopan); Boehringer Ingelheim GmbH, Ingelheim, Germany). Buscopan was administered by bolus injection immediately before starting the water enema using tap water $\left(2-3\right.$ litres, $\left.37^{\circ} \mathrm{C}\right)$. In cases where the abdominal PET/CT scan lasted more than 15 minutes, a short infusion of another $20 \mathrm{mg}$ of Buscopan dissolved in $50 \mathrm{ml}$ of sodium chloride $(0.9 \%)$ was administered over five minutes. PET/CT imaging from the diaphragm to the upper thighs was acquired with a limited breath-hold protocol in the prone position. ${ }^{29}$ Image acquisition was performed with $110 \mathrm{mAs}$,
$120 \mathrm{kV}, 3 \mathrm{~mm}$ slice thickness, and a $2.4 \mathrm{~mm}$ incremental reconstruction. CT acquisition started with a delay of 50 seconds after administration of $90 \mathrm{ml}$ of an iodinated contrast agent. PET imaging was acquired with the patient in the same position on the examination table covering the same field of view as CT. Acquisition time of PET was adapted in both parts according to the patient's weight: three minutes per bed position for patients weighing up to $65 \mathrm{~kg}$, 3.5 minutes for those weighing up to $85 \mathrm{~kg}$, and four minutes for those over $85 \mathrm{~kg}$

\section{Image evaluation}

Bowel distension was determined for each the following bowel segments according to a four point scale. The rectum was defined as ranging from the anorectal junction to the acetabular level. Subsequently, the sigmoid was defined as proximal to the rectum up to the level of the pelvic brim where the bowel did not re-enter the pelvis, the descending colon extended up to the splenic flexure, the transverse colon was found between the splenic and hepatic flexures, and the ascending colon was considered as the portion proximal to the midpoint of the hepatic flexure but distal to the ileocaecal valve. The caecum was proximal to the ileocaecal valve. The four point scoring system contained four grades of colonic distension: grade 0 , totally collapsed bowel; grade 1 , partially collapsed colon; grade 2, reasonable but suboptimally distended colon; and grade 3, optimal colonic distension with a barely visible colonic wall, according to previously published criteria. ${ }^{30}$ An average score for all bowel segments was calculated based on 84 measurements in total (six bowel segments each in 14 patients).

All CT and PET data sets were reviewed in fused and three dimensional modes on a dedicated workstation (Syngo Software; Siemens Medical Solutions). PET data sets were evaluated with and without attenuation correction. In cases where the anatomical situation remained unclear, three dimensional-multiplanar reformatting (3D-MPR) was performed. PET/CT data were evaluated by a radiologist and a nuclear medicine specialist in consensus (PET/CT experience: both 1.5 years). The evaluating physicians were informed about the clinical background, but blinded to the results of conventional colonoscopy. On PET/CT images, primary tumour assessment was based on detection of a contrast enhancing mass and/or thickened bowel wall. Focally increased glucose metabolism was defined as malignant on PET with a standard uptake value (SUV) exceeding 2.5 supporting the diagnosis. T staging was mainly based on the CT portion of the combined examination. Lymph nodes and distant organs were assessed for metastatic disease based on increased glucose metabolism with an SUV exceeding 2.5 (extrahepatic) and 3.5 (intrahepatic). Central necrosis was defined as an indicator of metastatic spread to lymph nodes independent of FDG uptake. Histopathological evaluation of the resected tumour and adjacent tissue served as the standard of reference in the 11 operated patients. Based on the small number of patients included in this feasibility study, no assessment of sensitivities and specificities was performed.

\section{RESULTS}

\section{Feasibility of imaging protocol}

PET/CT with integrated colonography provided fully diagnostic colonography data and whole body tumour staging in all patients. Bowel distension with water was feasible and showed good results based on the four grade scale. Mean average score for the rectum was 3.0, and for the sigmoid and descending colon mean distension was rated as 2.5 (fig 1A). Average scores for the transverse colon and ascending colon were 2.7 and the average score for the caecum was 2.6. There 


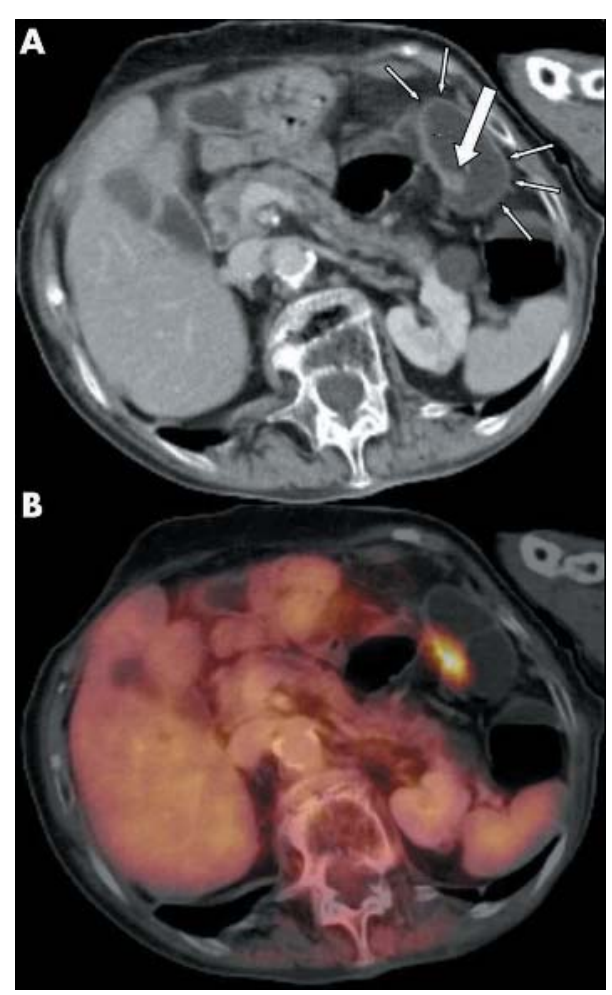

Figure 1 (A) Axial contrast enhanced computed tomography (CT) image demonstrated a tubulous polyp (diameter $9 \mathrm{~mm}$ ) at the left colon flexure (large white arrow). The colon was well distended, rated as distension grade 3 according to the four point scale (small white arrows). (B) Corresponding axial contrast enhanced positron emission tomography/CT revealed elevated glucose metabolism within this polyp and the adjacent bowel wall, indicating a carcinoma. Verification of an adenomatous carcinoma by histopathology followed.

were no complications in any of the 14 patients. All patients tolerated the procedure well, which included rectal water filling with prior pharmacological bowel relaxation and simultaneous negative oral contrast media administration for small bowel distension. The average time for the combined examination was 29 ( $\pm 6 \mathrm{~min}$ ) minutes, representing 6-10 bed positions per examination.

\section{PET/CT colonography}

PET/CT colonography and conventional colonoscopy revealed 18 colonic tumour sites in the group of 14 patients. There were, however, discrepancies between PET/CT colonography and colonoscopy in two patients. Conventional colonoscopy detected one additional $5 \mathrm{~mm}$ flat based polyp in a patient with a large tumour in the ascending colon. While the large tumour was detected on PET/CT colonography, PET/CT colonography missed the small polyp. Surgery revealed a benign polyp directly adjacent to the large tumour in the ascending colon. The polyp was of no clinical relevance as it was found to be a tubulous adenoma and within the surgical margins of the colonic carcinoma when resected. PET/CT colonography detected one additional colorectal tumour in the ascending colon which had been missed by colonoscopy. This was related to one incomplete colonoscopy procedure due to a stenotic and impassable lesion in the descending colon (fig 2). Histopathology of this lesion after resection proved this tumour to be malignant.

Four of the 11 patients undergoing tumour resection were found to be $\mathrm{N}$ positive while seven patients were $\mathrm{N}$ negative. PET/CT correctly classified $\mathrm{N}$ disease in $\mathrm{N}$ positive versus $\mathrm{N}$ negative in nine patients; no patient was overstaged and two

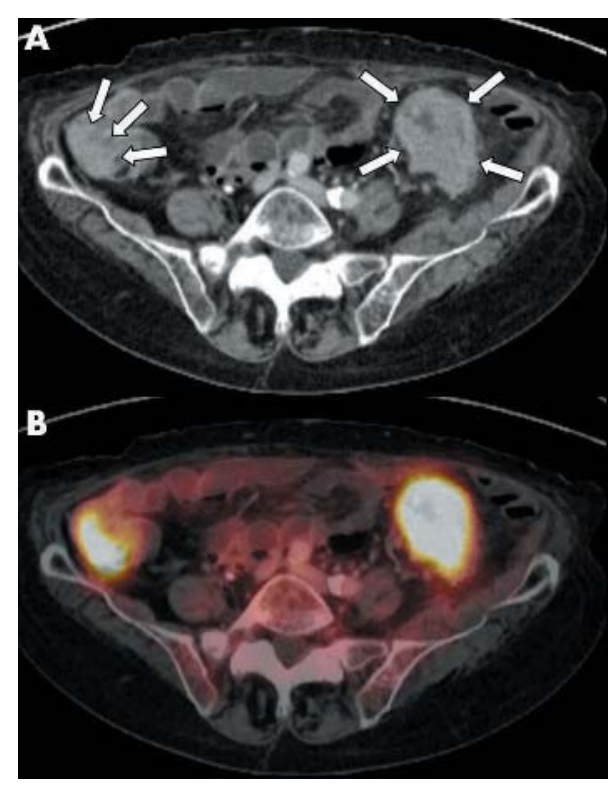

Figure 2 (A) Axial contrast enhanced computed tomography (CT) image showed one stenotic tumour site in the descending colon (four white arrows). This lesion was impassable during conventional colonoscopy. Another tumorous mass was found on the ascending colon (three white arrows), later proven to be malignant by histopathology. (B) Axial contrast enhanced positron emission tomography/CT (PET/CT) colonography revealed both tumour sites with elevated glucose metabolism and clear circumscription. PET/CT also indicated tumorous infiltration of the adjacent tissue. This was later verified by histopathology.

patients were understaged. One $3 \mathrm{~mm}$ lymph node metastasis was not detected due to normal glucose metabolism. Another lymph node metastasis was missed based on its proximity to the primary lesion. In the other two patients with lymph node metastases, the functional information provided by PET/CT proved valuable for detection of tumour harbouring lymph nodes (fig 3).

According to the standard of reference, $\mathrm{T}$ stage was $\mathrm{T} 0$ in one patient, $\mathrm{T} 1$ in one patient, $\mathrm{T} 2$ in one patient, $\mathrm{T} 3$ in six patients, and T4 in two patients. Nine patients in whom the tumour was resected showed G2 and two patients showed G3 grading. Whole body PET/CT colonography correctly classified the $\mathrm{T}$ stage in $8 / 11$ resected patients (fig 4 ). Of these eight correctly diagnosed patients, one patient had a Tl lesion, two patients were $\mathrm{T} 2$, seven patients were $\mathrm{T} 3$, and one patient was T4. One patient showed a tubulous $9 \mathrm{~mm}$ polyp with increased glucose metabolism at the left colonic flexure. Based on increased glucose metabolism, the PET/CT readers suggested malignancy (fig 1). Histopathology from the resected specimen verified malignant tumour growth. However, PET/CT demonstrated increased glucose metabolism suggesting malignancy in one patient with three tumour sites, but histopathology showed high grade intraepithelial dysplasia without cancerous growth.

\section{Whole body staging}

In total, six additional extracolonic tumour sites were detected with PET/CT in six patients; five tumour sites were unknown. These included three patients with liver metastases, one patient with additional breast cancer, a patient with a synchronous hepatocellular carcinoma and pulmonary metastases, as well as one patient with thyroid carcinoma. Histological verification of extracolonic findings was obtained for the patients with breast cancer, hepatocellular carcinoma, and thyroid gland carcinoma. The other tumour sites were verified by clinical follow up. 


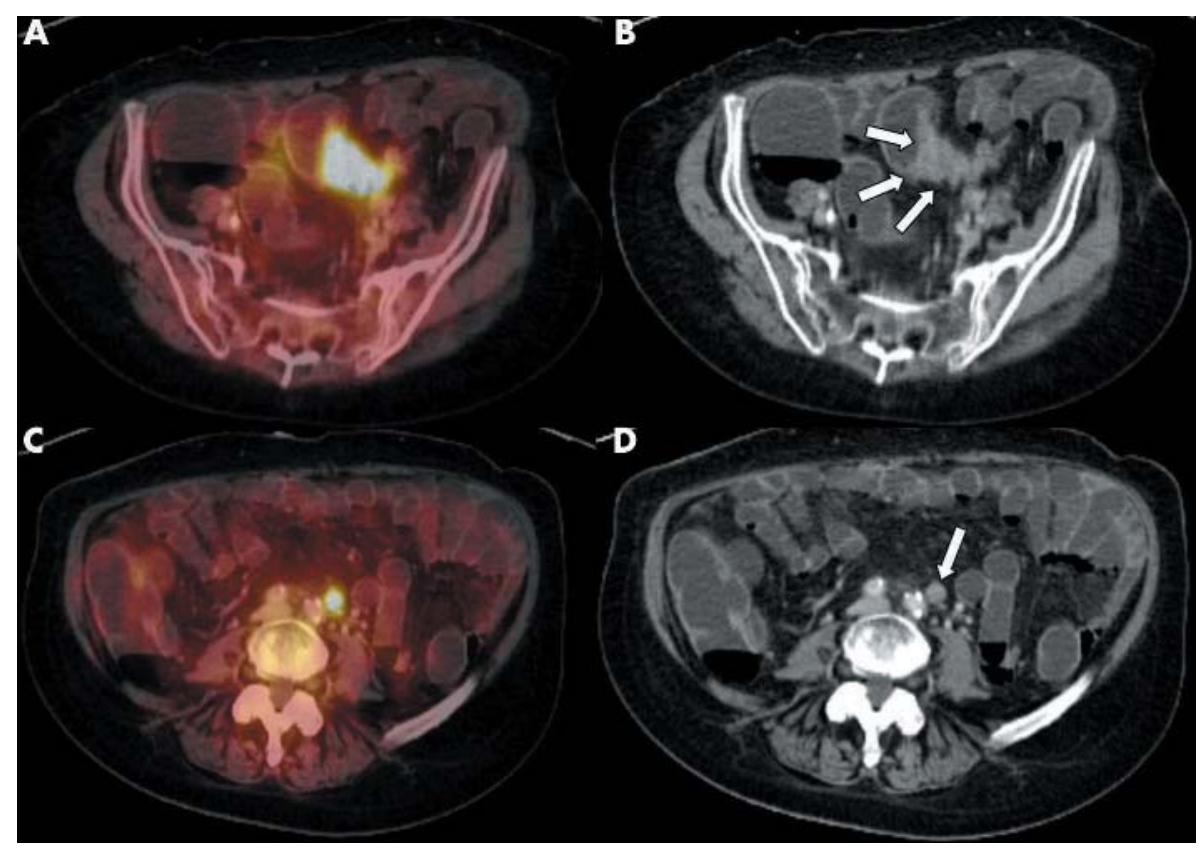

Figure 3 (A, B) Axial contrast enhanced positron emission tomography/computed tomography (PET/CT) and CT demonstrated a mass in the transverse colon without infiltration of the adjacent tissue (white arrows). (C, D) In the same patient, PET/CT detected elevated glucose metabolism in a lymph node (C) characterising it as malignant. On CT the lymph node was considered suspicious based on its round shape but it was not pathologically enlarged. Histopathology revealed metastatic spread.

\section{DISCUSSION}

Whole body PET/CT colonography demonstrated high detection rates for colonic lesions as well as abdominal lymph nodes. Additionally, M stage was accurately assessed by the integrated whole body protocol. Therefore, whole body PET/ CT colonography may play an important role as an "all in one" staging modality in patients with colorectal cancer,

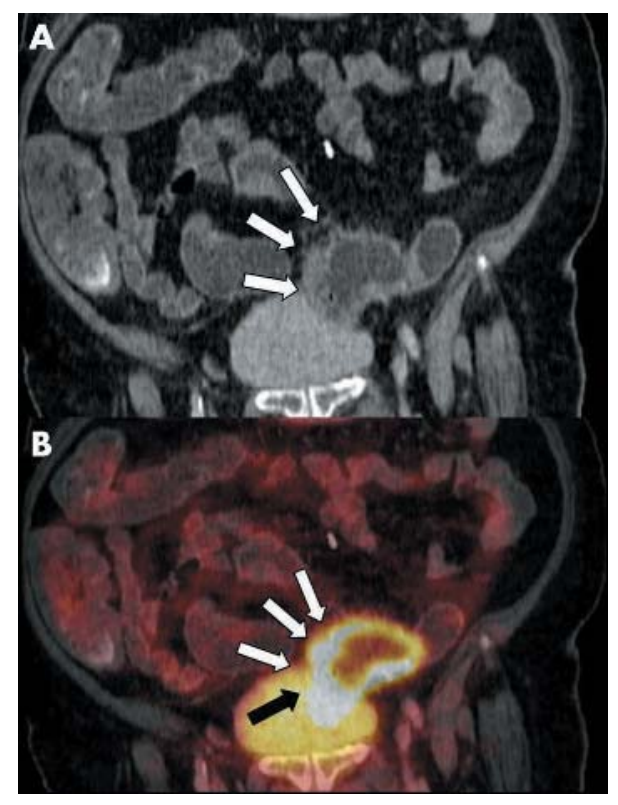

Figure 4 (A) Coronal contrast enhanced computed tomography (CT) image showed thickened sigmoid wall with suspected infiltration of the surrounding tissue (white arrows). (B) Coronal contrast enhanced positron emission tomography/CT demonstrated elevated glucose metabolism within the thickened bowel wall and indicated infiltration of the surrounding fat tissue (white arrows) as well as urinary bladder infiltration in the dorsal bladder wall (black arrow), which had not been detected on CT. especially those patients with incomplete optical colonoscopy or questionable synchronous lesions.

Several issues need to be addressed when applying a dedicated CT colonography protocol for cancer staging to a single whole body PET/CT examination. In general, image misregistration due to bowel movement must be considered problematic as the time between acquisition of $\mathrm{CT}$ and the end of PET acquisition is approximately 15 minutes. ${ }^{31}$ To avoid bowel movement during this time period, bowel relaxation has to be extended over a longer period of time compared with conventional CT colonography protocols. Comparable with conventional CT staging, the PET/CT protocol uses an intravenous bolus injection of $20 \mathrm{mg}$ N-butylscopolamine (Buscopan) before the start of the examination. ${ }^{7}$ However, if the time between CT and PET outlasts the half life of $\mathrm{N}$-butylscopolamine, additional bowel relaxation is required. This was given by short infusion of an additional $20 \mathrm{mg}$ of Buscopan. In total, two thirds of the maximal allowed daily dose of Buscopan was given. This protocol resulted in exact coregistration in nearly all colon segments. As N-butylscopolamine is not FDA approved in the USA, studies evaluating the use of glucagon in this modified and optimised protocol are required.

A practical challenge in CT colonography is patient scanning in both the prone and supine positions, which has been shown to provide better colonic distension than either patient position alone. Apart from the additional radiation exposure, imaging the patient twice with PET and CT substantially lengthens the time for abdominal image acquisition. In addition, previous studies comparing image acquisition in the prone and supine positions versus only one position did not detect an increase in sensitivities for polyp detection. ${ }^{32}$ Thus image acquisition was limited to the prone position in the PET/CT protocol.

A different approach in colonic CT scanning is the use of inflating room air or $\mathrm{CO}_{2}$ which has been shown to provide improved patient comfort due to bowel absorption of the gas. ${ }^{70} 1132$ However, colonic distension by $\mathrm{CO}_{2}$ or room air inflation is impaired by the need for additional air inflation 
during the procedure based on intestinal absorption of the gas. We considered this option to be less effective in our setting as absorption of the gas between acquisition of CT and PET would possibly lead to differences in bowel distension, resulting in image misregistration of morphological and functional data. Furthermore, additional air inflation may result in bowel movement, increasing the amount of image misregistration. Thus rectal water filling was chosen for bowel distension.

CT colonography protocols are often performed with multidetector CT as well as different post-examination software applications, such as virtual colonoscopy. ${ }^{33-35}$ To date, there is no equivalent PET/CT software available. CT images of combined PET/CT can be viewed separately applying these software tools but there is no software available providing virtual colonoscopy of the fused data sets. Therefore, image evaluation was limited to fused axial as well as 3D-MPR images in this study. Despite the fact that multidetector scanners provide the option of acquiring thinner slices with corresponding reconstruction increments, it already has been shown that there may not be a significant difference in detection of small intestinal masses between scanning with single detector or multidetector scanners. ${ }^{36}$ Further studies comparing dual slice PET/CT with the new 16 or 64 slice PET/CT generation for PET/CT colonography will address this issue.

While the results of this study must be interpreted with caution because of the small sample size, they do demonstrate a trend towards accurate staging results with this dedicated whole body PET/CT colonography protocol. In addition, PET/CT revealed one patient harbouring three colonic masses, and increased glucose metabolism indicated malignancy in these sites. As histopathology found severe intraepithelial dysplasia but no malignancy, these must be considered false positives. However, it is well known that intraepithelial dysplasia may result in cancerous growth. Thus detection of these lesions must be considered relevant. Based on the reported results, the dedicated PET/CT colonography protocol may also be eligible for detection of dysplastic tissue even before cancerous transformation. Further studies addressing this issue are required.

While there is only a small number of studies on FDG-PET/ $\mathrm{CT}$ in colon cancer, significantly more accurate staging results for $\mathrm{T}$ and $\mathrm{N}$ stages have been reported for other tumours when staged with PET/CT compared with CT alone and PET alone. ${ }^{1522} 2627$ In colon cancer patients, PET may be used for staging of the primary tumour and for diagnosis and staging of tumour recurrence. Although superior results have been published in terms of sensitivity and specificity for PET alone over CT alone when assessing the $\mathrm{M}$ stage for colorectal cancer, PET imaging as the sole procedure cannot be considered an option as additional morphological information is required for lesion localisation. Side by side image evaluation of separately acquired CT and PET data sets may be an option. However, substantial misregistration of the bowel when correlating CT with PET must be expected due to patient movement between the two procedures.

The question to be answered in future analyses will be whether full dose CT data are required for the combined protocol of whole body PET/CT and PET/CT colonography. To date, different CT colonography protocols have been reported in the literature, which are based on different radiation doses depending on the required information. It has however already been shown that in CT colonography and CT staging, contrast material and full dose image acquisition can increase lesion detection rates. ${ }^{8}{ }^{11} 1237$ PET/CT tumour staging may also benefit from full dose and contrast enhanced CT. Apart from the sensitivity of lesion detection, issues such as the accuracy of $\mathrm{T}$ and $\mathrm{N}$ staging, radiation exposure, and potential adverse reactions to the iodine based contrast material have to be considered.

A limitation of the combined PET/CT protocol is the time required for image acquisition. Compared with dedicated CT colonography protocols, examination times of up to $30 \mathrm{~min}$ utes are substantially longer. However, examination times are similar compared with whole body PET/CT without colonography. In the future, the examination time of whole body PET/CT colonography may be substantially lowered by development of alternative PET detector materials and the introduction of new PET detectors covering a larger field of view. ${ }^{38}$

In conclusion, whole body PET/CT with an integrated PET/ CT colonography protocol is technically feasible for staging colorectal cancer. PET/CT colonography will not compete with colonoscopy, rather the two procedures will complement one another. Patients with suspected colorectal cancer but incomplete colonoscopy may substantially benefit from the combined imaging protocol. Synchronous tumours and precancerous polyps may alter surgery to a more tailored approach. In cases with incomplete colonoscopy, these tumours can be detected by PET/CT colonography. Additionally, in comparison with CT colonography, small colonic lesions may be identified more easily based on the additional functional data. However, this study did not directly compare PET/CT with CT colonography. Thus this question will have to be answered in further studies. A major advantage of the combined imaging approach is to the fact that the diagnosis of the primary tumour as well as whole body $\mathrm{N}$ and $\mathrm{M}$ staging can be performed in a single examination session. The typical multimodality multi-session staging procedure may eventually be replaced by one optimised whole body examination. As the number of patients is too small to draw definitive conclusions on accuracy, this study will hopefully encourage further investigations addressing the overall accuracy of whole body PET/ CT colonography for TNM staging.

\section{Authors' affiliations}

P Veit, C Kühle, H Kuehl, C U Herborn, J Barkhausen, G Antoch, Department of Diagnostic and Interventional Radiology and

Neuroradiology, University Hospital Essen, Essen, Germany

T Beyer, H Stergar, A Bockisch, Department of Nuclear Medicine, University Hospital Essen, Essen, Germany

G Börsch, Department of Internal Medicine, Elisabeth Hospital Essen, Essen, Germany

Conflict of interest: None declared.

\section{REFERENCES}

1 Jemal A, Tiwari RC, Murray T, et al. Cancer statistics, 2004. CA Cancer J Clin 2004;54:8-29.

2 Jemal A, Murray T, Samuels A, et al. Cancer statistics, 2003. CA Cancer J Clin 2003;53:5-26.

3 Jemal A, Thomas A, Murray T, et al. Cancer statistics, 2002. CA Cancer J Clin 2002;52:23-47.

4 Gore RM. Colorectal cancer. Clinical and pathologic features. Radiol Clin North Am 1997;35:403-29.

5 Bipat S, Glas AS, Slors FJ, et al. Rectal cancer: local staging and assessment of lymph node involvement with endoluminal US, CT, and MR imaging - a metaanalysis. Radiology 2004;232:773-83.

6 Stevenson GW. Colorectal cancer imaging: a challenge for radiologists. Radiology 2000;214:615-21.

7 Neri E, Giusti P, Battolla L, et al. Colorectal cancer: role of CT colonography in preoperative evaluation after incomplete colonoscopy. Radiology
2002;223:615-19.

8 Filippone A, Ambrosini R, Fuschi $\mathrm{M}$, et al. Preoperative $\mathrm{T}$ and $\mathrm{N}$ staging of colorectal cancer: accuracy of contrast-enhanced multi-detector row CT colonography-initial experience. Radiology 2004;231:83-90.

9 Saunders TH, Mendes Ribeiro HK, Gleeson FV. New techniques for imaging colorectal cancer: the use of MRI, PET and radioimmunoscintigraphy for primary staging and follow-up. Br Med Bull 2002;64:81-99.

10 Yee J, Akerkar GA, Hung RK, et al. Colorectal neoplasia: performance characteristics of CT colonography for detection in 300 patients. Radiology 2001;219:685-92. 
11 Morrin MM, Farrell RJ, Kruskal JB, et al. Utility of intravenously administered contrast material at CT colonography. Radiology 2000;217:765-71.

12 Sosna J, Morrin MM, Kruskal JB, et al. Colorectal neoplasms: role of intravenous contrast-enhanced CT colonography. Radiology 2003;228: 152-6.

13 Sosna J, Morrin MM, Kruskal JB, et al. CT colonography of colorectal polyps: a metaanalysis. AJR Am J Roentgenol 2003;181:1593-8.

14 Hara AK, Johnson CD, MacCarty RL, et al. Incidental extracolonic findings at CT colonography. Radiology 2000;215:353-7.

15 Antoch G, Vogt FM, Freudenberg LS, et al. Whole-body dual-modality PET/ $\mathrm{CT}$ and whole-body MRI for tumor staging in oncology. JAMA 2003;290:3199-206

16 Rohren EM, Turkington TG, Coleman RE. Clinical applications of PET in oncology. Radiology 2004;231:305-32.

17 Kantorova I, Lipska L, Belohlavek O, et al. Routine (18)F-FDG PET preoperative staging of colorectal cancer: comparison with conventional staging and its impact on treatment decision making. J Nucl Med 2003;44:1784-8

18 Valk PE, Abella-Columna E, Haseman MK, et al. Whole-body PET imaging with[18F]fluorodeoxyglucose in management of recurrent colorectal cancer. Arch Surg 1999;134:503-11.

19 Abdel-Nabi H, Doerr RJ, Lamonica DM, et al. Staging of primary colorectal carcinomas with fluorine-18 fluorodeoxyglucose whole-body PET: correlation with histopathologic and CT findings. Radiology 1998;206:755-60.

20 Kalff V, Hicks RJ, Ware RE, et al. The clinical impact of (18)F-FDG PET in patients with suspected or confirmed recurrence of colorectal cancer: a prospective study. J Nucl Med 2002;43:492-9.

21 Huebner RH, Park KC, Shepherd JE, et al. A meta-analysis of the literature for whole-body FDG PET detection of recurrent colorectal cancer. J Nucl Med 2000;41:1177-89.

22 Cohade C, Osman M, Leal J, et al. Direct comparison of (18)F-FDG PET and $\mathrm{PET} / \mathrm{CT}$ in patients with colorectal carcinoma. J Nucl Med 2003:44:1797-803.

23 Beyer T, Townsend DW, Blodgett TM. Dual-modality PET/CT tomography for clinical oncology. Q J Nucl Med 2002;46:24-34

24 Beyer T, Townsend DW, Brun T, et al. A combined PET/CT scanner for clinical oncology. J Nucl Med 2000;41:1369-79.

25 Bar-Shalom R, Yefremov N, Guralnik L, et al. Clinical performance of PET/CT in evaluation of cancer: additional value for diagnostic imaging and patient management. J Nucl Med 2003;44:1200-9.
26 Antoch G, Stattaus J, Nemat AT, et al. Non-small cell lung cancer: dual-modality PET/CT in preoperative staging. Radiology 2003;229:526-33

27 Even-Sapir E, Parag Y, Lerman $\mathrm{H}$, et al. Detection of recurrence in patients with rectal cancer: PET/CT affer abdominoperineal or anterior resection. Radiology 2004;232:815-22.

28 Antoch G, Kuehl H, Kanja J, et al. Dual-modality PET/CT scanning with negative oral contrast agent to avoid artifacts: introduction and evaluation. Radiology 2004;230:879-85.

29 Beyer T, Antoch G, Blodgett T, et al. Dual-modality PET/CT imaging: the effect of respiratory motion on combined image quality in clinical oncology. Eur J Nucl Med Mol Imaging 2003;30:588-96.

30 Taylor SA, Halligan S, Goh V, et al. Optimizing colonic distention for multidetector row CT colonography: effect of hyoscine butylbromide and rectal balloon catheter. Radiology 2003;229:99-108.

31 Nakamoto $\mathrm{Y}$, Chin BB, Cohade C, et al. PET/CT: artifacts caused by bowel motion. Nucl Med Commun 2004;25:221-5.

32 Yee J, Kumar NN, Hung RK, et al. Comparison of supine and prone scanning separately and in combination at CT colonography. Radiology 2003;226:653-61

33 Fenlon HM, Nunes DP, Schroy PC 3rd, et al. comparison of virtual and conventional colonoscopy for the detection of colorectal polyps. N Engl J Med 1999;341:1496-503.

34 Pickhardt PJ, Choi JR, Hwang I, et al. Nonadenomatous polyps at CT colonography: prevalence, size distribution, and detection rates. Radiology 2004;232:784-90

35 Edwards JT, Mendelson RM, Fritschi L, et al. Colorectal neoplasia screening with CT colonography in average-risk asymptomatic subjects: communitybased study. Radiology 2004;230:459-64.

36 Hara AK, Johnson CD, MacCarty RL, et al. CT colonography: single- versus multi-detector row imaging. Radiology 2001;219:461-5.

37 Fletcher JG, Johnson CD, Krueger WR, et al. Contrast-enhanced CT colonography in recurrent colorectal carcinoma: feasibility of simultaneous evaluation for metastatic disease, local recurrence, and metachronous neoplasia in colorectal carcinoma. AJR Am J Roentgenol 2002; 178:283-90.

38 Halpern BS, Dahlbom M, Quon A, et al. Impact of patient weight and emission scan duration on PET/CT image quality and lesion detectability. J Nucl Med 2004;45:797-801

\title{
EDITOR'S QUIZ: GI SNAPSHOT
}

\begin{abstract}
Answer
From question on page 53

Magnetic resonance (MRI) axial and coronal T2 weighted images (fig 1A, B) showed ascites, extensive liver and kidney cysts, and narrowing of the cava vein. MRI gadolinium enhanced Tl weighted image (fig lC) confirmed the cystic nature of the liver lesions without enhancement. Sagittal plane reconstruction of the image (fig ID) demonstrated severe compression of the retrohepatic cava vein (arrow) that was confirmed by the cavogram (fig 1E), also depicting large paravertebral collateral veins. Autosomal dominant polycystic kidney/liver disease causing compression of the vena cava was diagnosed.

A Palmaz stent was placed in the narrowed vein. In the following days, daily urine volume increased and, at discharge, furosemide $75 \mathrm{mg}$ /daily was prescribed. The patient regained body weight and a good quality of life; after 66 months of follow up she remains well and ultrasonography shows only minimal ascites.

Caval hypertension may have a relevant role in determining ascites accumulation in patients with polycystic liver and therefore in this setting inferior vena cava patency should be accurately evaluated. MRI is a useful diagnostic tool and, if stenosis is showed, caval stenting should be the first therapeutic procedure.
\end{abstract}

doi: $10.1136 /$ gut.2005.067868 